
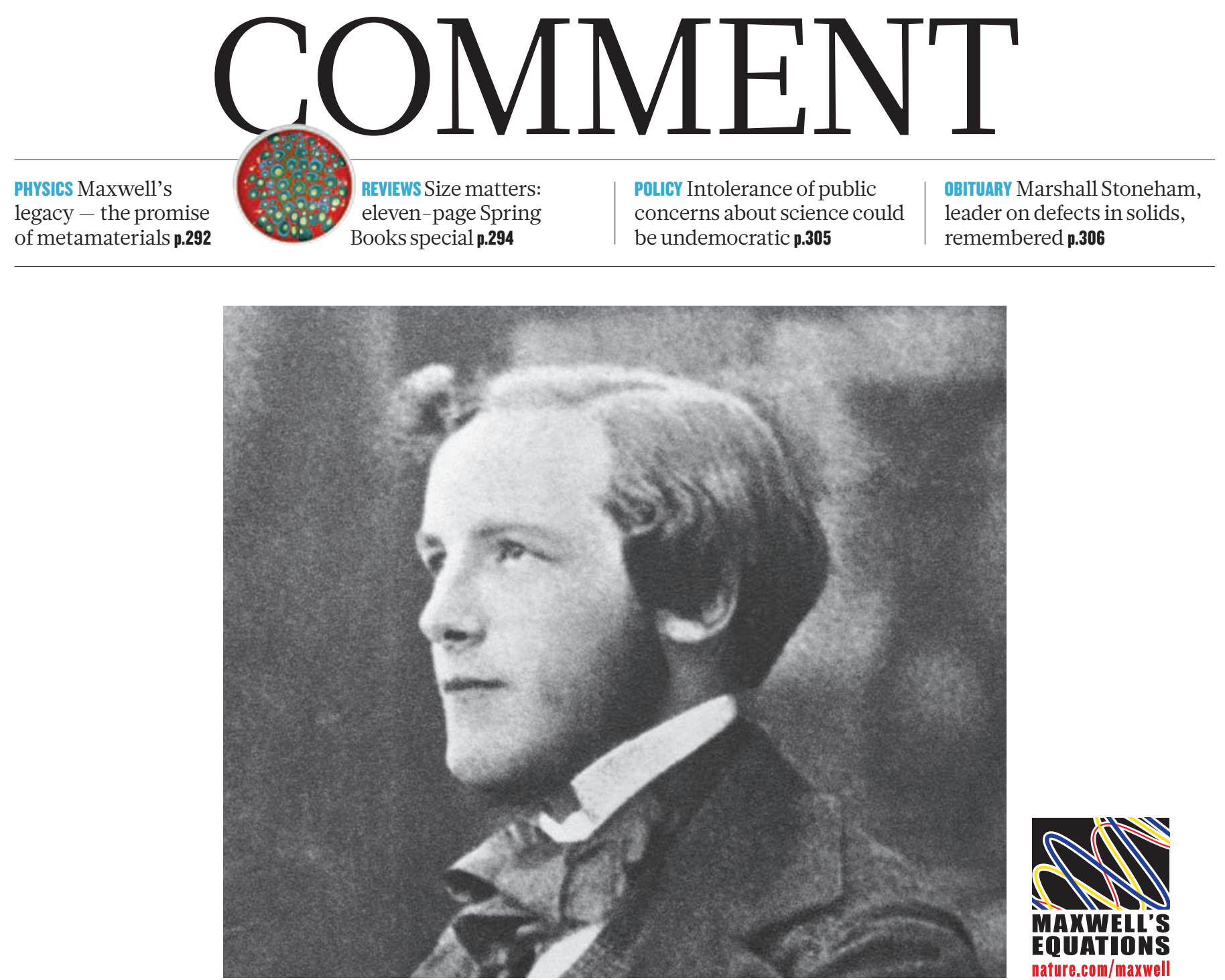

As a young scientist, Maxwell wrote down the equations that laid the foundations of modern physics.

\title{
The laird of physics
}

\section{James Clerk Maxwell's 1861 work on electromagnetism, which unified scientific fields, was driven as much by technology as by abstract theorizing, argues Simon Schaffer.}

$\mathrm{O}$ ne hundred and fifty years ago this month, the 30 -year-old James Clerk Maxwell published the first part of an extraordinary document: On Physical Lines of Force, a paper on the mathematical physics of electromagnetism. It is tempting to find in it much familiar furniture of the modern world: telecommunications, information technology and microelectronics. But the path from then to now is not as straightforward as it may seem. Maxwell's admirers sometimes treat his work as evidence that disinterested science eventually delivers huge economic and practical pay-offs. In the United Kingdom, for instance, 'Clerk Maxwell' has been proposed as the brand name for publicly funded regional centres for technology and innovation, with the ambitious goal of closing the gap between university and industry.

This linear model - the idea that cloistered theorizing can lead directly to new technology and profits - is often used by those arguing for greater investment in science. But a 2010 report from Britain's Royal Society titled The Scientific Century notes that "the linear model of basic research through to innovation bears little relation to reality". It adds that the model's long-lasting appeal is partly explained by historical assumptions and familiar myths that are rarely challenged. Maxwell's magnificent work of the $1860 \mathrm{~s}$ is an excellent example. Rather than a stately progression from abstract theory to solid application, it was the product of a web of markets, technologies, labs and calculators in the workshop of the world.

\section{MYTHS AND MATHEMATICS}

Why was Maxwell even working on electromagnetism? In the 1850s it was not common fodder for mathematicians educated at the University of Cambridge. The university's curriculum focused on the established sciences of celestial mechanics, wave optics and hydrodynamics. British universities did not even have teaching laboratories for physics. It is telling that the best-known British pioneer of electromagnetic studies was the Royal Institution professor Michael 
- Faraday, a prodigious experimental chemist without formal education who knew little of higher mathematics.

Maxwell, unlike his hero Faraday, sometimes seemed a pastoral recluse. He often retreated to his 1,800-acre Scottish estate, where rural affairs absorbed much of his time - he was even described as a "north-country laird". This cultivated self-image seemed to support the folklore of the brilliant scientist divorced from mundane trade.

Yet as a bright teenager in Galloway, in southwest Scotland, Maxwell had been unusually keen on turning classroom principles into practical devices, including homebuilt electric telegraphs. With his father's earnest backing, Maxwell studied engines on show in the manufacturing towns and at the Great Exhibition of the Works of Industry of all Nations at the Crystal Palace in London in 1851. His ingenuity attracted the attention of William Thomson (later Lord Kelvin) - the two met in 1850. The young professor at the University of Glasgow encouraged Maxwell to manufacture magnetically sensitive crystals at home in Galloway.

After Maxwell completed his Cambridge mathematics training in early 1854, he understandably saw himself as "an electrical freshman". But Faraday's fascinating studies of electrical currents and magnetic poles drew his attention. So he asked Thomson, who had already been working on these puzzles, for guidance so that he and other graduates could at last "attack electricity". The timing was perfect.

\section{COMMUNICATION PROBLEMS}

A project launched in the 1850s, aiming to tie the British Empire together with a global electric telegraph system, posed many of the problems that Maxwell and his scientific allies had to solve: the propagation of signals in electromagnetic networks, the reliability of measuring instruments, the analysis of force, resistance and current. It is, perhaps, significant that electromagnetic field theory was invented in the nation most concerned with submarine telegraphs. Elsewhere in Europe, overhead lines were commonplace, so problems caused by sea water in distorting and delaying signal transmission were not evident.

In 1853, Faraday witnessed the catastrophic problems of signal delay in cable lines laid between London and Manchester by a telegraph company. He saw these troubles as a chance to publicize his theory of electromagnetic induction. In a Royal Institution lecture a few months later, Faraday used his theory to show how sea water would act on submarine cables like the outer casing of a condenser, increasing the cable's capacitance, slowing down induction and so delaying the signal even more.

Maxwell and Thomson closely studied Faraday's lecture - and the warnings it held for submarine telegraphy. Within months Thomson

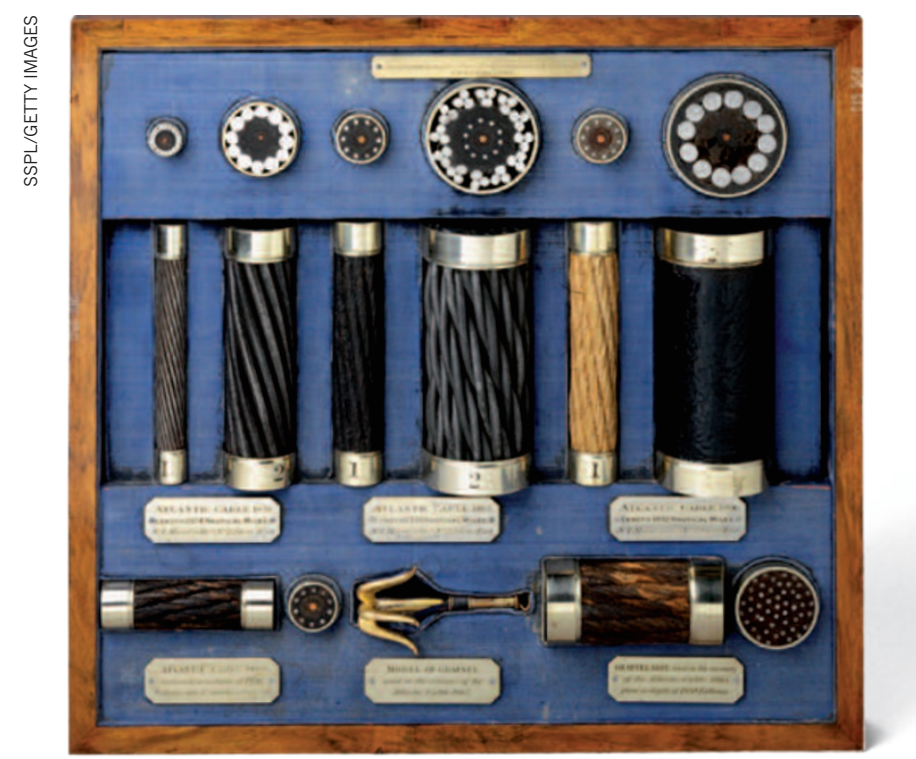

Sections of the Atlantic cables from 1858, 1865 and 1866.

had a formula for the relationship between cable length and signal delay, of much interest to investors backing an ambitious scheme for a 5,000-kilometre undersea cable to North America. By 1856, Thomson had become a director of a major transatlantic cable company and, from his new Glasgow physics lab, a recognized authority on electromagnetic systems. In subsequent years, he earned many thousands of pounds from telegraph patents and consultancies, more than enough to buy a yacht, build a baronial mansion and endow his university with fellowships and equipment. Maxwell greatly admired Thomson for "bringing his obtrusive science to bear upon the engineers".

Maxwell meanwhile, now a professor of natural philosophy in Aberdeen, boldly reworked Faraday's model of electromagnetic induction. He reckoned that Faraday had destroyed fashionable continental models of electromagnetism, which posited instant actions across empty spaces between isolated particles. Maxwell began to see electromagnetism as dependent on some kind of fluid medium, a space-filling ether or field whose tension and stress stored energy and transmitted action at finite speeds. It took Maxwell four challenging

"British natural philosophers were trained to think in terms of the physics of pulleys, pumps, jelly or rubber." years from spring 1857 to make this model of electromagnetism match the best available data, first in Scotland, then at his new job as chair of natural philosophy at King's College London. British natural philosophers, unlike their contemporaries in France and Germany, were trained to think in terms of the physics of pulleys, pumps, jelly or rubber, then extended to the Universe. This was the approach Maxwell had learned in his studies of fluid mechanics at Cambridge. And so his paper of spring 1861 published in The London, Edinburgh and Dublin Philosophical Magazine and Journal of Science pictured the space of Faraday's magnetic lines of force as a vast array of spinning gears separated by long strings of ball-bearings.

\section{MECHANICAL EQUATIONS}

Within this 1861 paper can be found, distributed through different sections of his argument, Maxwell's first versions of the equations that characterize the electromagnetic field. Yet it was only much later that the equations were identified as fundamental building blocks of a new electromagnetic system. They summarized principles established by European experimenters in the previous decades. Electric flux through a closed surface is proportional to the electric charge it encloses; magnetic poles cannot exist in isolation; the electric current along a closed loop is related to the magnetic field round the loop; electromagnetic induction varies with time. To these principles Maxwell added the notion of a displacement current, generated even in apparently empty space by changing electrical forces.

In his 1861 model, Maxwell showed that, when suitably adjusted, the workings of his complex machinery of gears and wheels matched those of electromagnetism. Magnetic energy was stored in the kinetic energy of the spinning gears, and the gears would spin and bulge, capturing the way changes in magnetic fields generated currents. With these achievements in print, Maxwell seemed entirely content when he went off to his country estate in summer 1861. In the next few months, however, his understanding of the significance of his own work changed significantly.

Over that summer, telegraph problems preoccupied British physicists. In the wake of the disastrous first attempt to lay a transatlantic cable, the British Association for the Advancement of Science set up a committee to determine standards of electrical resistance to make the cable system work. Within a year of the committee's formation, Maxwell had joined enthusiastically, and electromagnetic measurements started to dominate Britain's new physics teaching laboratories, including the one run by Thomson at Glasgow and, eventually, the Cavendish Laboratory led by Maxwell at Cambridge.

In Scotland in the summer of 1861, Maxwell thought harder about his model of fluid gears and wheels. Accurate technical measurements dictated some of the parameters of its structure. Maxwell realized 


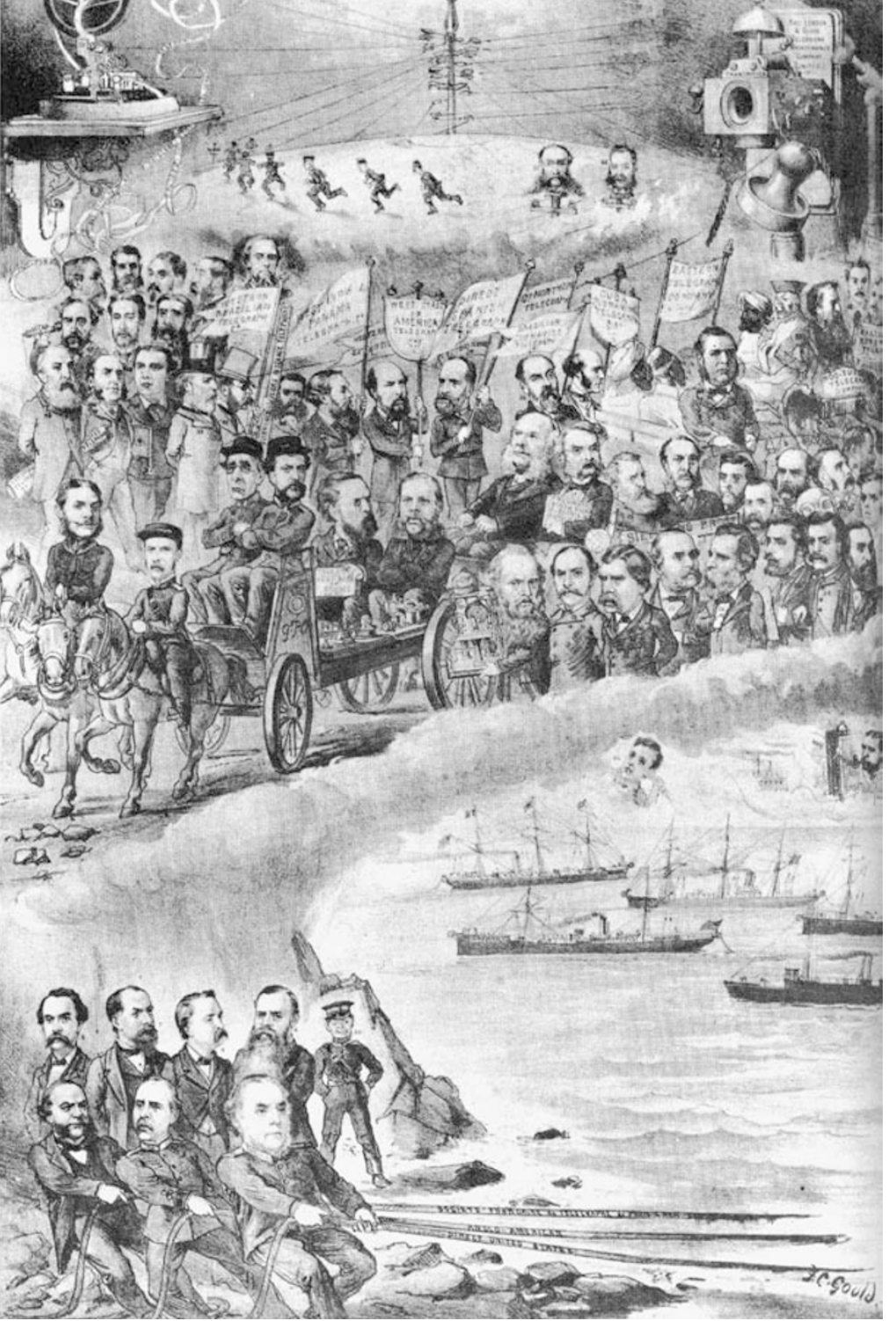

An 1883 cartoon pictures physicists, engineers and bankers side by side.

that his fluid medium must transmit actions through transverse waves, just like rubber or jelly. He could calculate the waves' velocity from the density and rigidity of the medium - determined by practical laboratory measures of the electrostatic and electromagnetic forces. Back in London, he checked electromagnetic observations made in the late 1850s in Germany and plugged these values into his equations. To his apparent surprise, the ratio of the electrostatic and electromagnetic units differed by no more than about $1 \%$ from the value of light speed measured in Paris a decade earlier. "The coincidence is not merely numerical," he told Faraday in autumn 1861. It seemed that light was thus a vibration in the medium responsible for electromagnetism. With this insight, Maxwell launched the unification of the apparently disparate fields of light and electromagnetism and helped to change physics forever.

The reception of Maxwell's discoveries was also influenced by practical concerns with telegraphic signalling. In the amazingly fertile period before his early death in 1879, Maxwell strove to turn his mechanical model of the electromagnetic field into a generalized dynamic theory of electromagnetic action. Despite its central importance to physics and the modern understanding of all types of radiation, Maxwell's theory was slow to catch on.

One of its sternest critics was none other than Thomson. He never really forgave Maxwell for replacing the language of complex machinery with an abstract algebraic notation. He saw Maxwellian electromagnetism as "rather a backward step", if not a kind of "nihilism". One reason the two great scientists differed was because, from the first, they had different understandings of telegraph signalling. Maxwell held that Faraday's initial investigation of signal distortion needed a unified treatment of induction, conduction and the surrounding medium. Thomson's models of telegraphy relied on separate analyses of conduction along the wires and induction through the insulator round them.

In 1884, five years after Maxwell's death, Thomson even publicly claimed that he'd known all about the electromagnetic theory of light long before, because of his analysis of signal propagation in submarine cables. It seemed Thomson was confusing light speed in the electromagnetic field, Maxwell's great topic, with signal speed in an underwater cable, Thomson's specialty. The Irish physicist George Francis Fitzgerald, one of Maxwell's young disciples, had to write in protest to Nature "to prevent what I find is a very common mistake". The link between telegraph engineering and the fate of electromagnetic theory lasted beyond Maxwell's lifetime.

In the preface to his 1873 masterpiece, A Treatise on Electricity and Magnetism, Maxwell explained his debt to telegraphy, arguing that it gave "a commercial value to accurate electrical measurements" and also allowed tests "on a scale which greatly transcends that of any ordinary laboratory", private or public. Indeed, telegraphy provided Maxwell with crucial support for his theory through measures of the electrostatic and electromagnetic units made in 1868-69.

Maxwell's decision to take over Cambridge's new experimental physics lab was in part driven by the need for such engineering resources. Happily, by the 1870 s, Cambridge mathematics teachers were introducing students to submarine telegraphy in their new course on Maxwellian electromagnetism.

\section{ORDINARY GROWTH}

In sum, On Physical Lines of Force is an odd text to use as example of the unyielding purity of physical science. Maxwell's formulae did not appear in their most familiar form until almost 25 years after its publication. The four famous equations linking electromagnetic forces and fluxes owe their elegant and economical vector form to a brilliant London telegraphist, Oliver Heaviside. He published them in 1885 in The Electrician, a trade journal for electrical engineers and businessmen.

A veteran of the 1860 s submarine cable schemes, Heaviside needed mathematical tools for managing energy transmission without distortion or loss. Unknown to the solitary telegraphist, a young German physicist, Heinrich Hertz, also established these simplified expressions for the field equations at the same time. Practical problems helped make these beautiful expressions, even in their most familiar, and apparently most abstract, mathematical form. For some time after 1885, they were not the Maxwell but the Hertz-Heaviside equations.

The French scientist Pierre Duhem saw this new physics as a sign of the rosbifs' industrial utilitarianism. "We thought we were entering the tranquil and neatly ordered abode of reason," Duhem complained of a Maxwellian textbook, "but we find ourselves in a factory." This was satirical, but in many ways it was spot on. There was an intimate and complex relation between the interests of scientific inquiry and commercial, industrial and technical enterprises. Victorians knew that well.

An 1883 cartoon (pictured), in a popular financial magazine, showed British physicists marching side by side with bankers, engineers, publishers and stockbrokers. Over the caption "telegraph and telephone mag(net)ates", the cartoon targeted the threat of Thomas Edison's new telephone to the cable system. In the last public lecture he gave, Maxwell showed a University of Cambridge audience this telephone. He had a performance of the song Men of Harlech transmitted from the Sedgwick Museum of Earth Sciences across town to the Senate House. And he taught the key lesson that the linkage of "these currents of the telephone which produce an audible effect" with precision electromagnetism was part of "the ordinary growth of scientific principles". Maxwell's triumphs of 150 years ago still have much to teach us about how this 'ordinary growth' works.

Simon Schaffer is in the Department of History and Philosophy of Science, University of Cambridge, Cambridge CB2 3RH, UK. e-mail:sjs16@hermes.cam.ac.uk 